\title{
The Ability of Novel Urinary Biomarkers (NGAL and IL-18) in Predicting the in-Hospital Mortality, the Need of Renal Replacement Therapy and the Future Development of Chronic Kidney Disease in Post-Cardiac Sur- gery Acute Kidney Injury Patients: A New Horizon of Hope on the Anvil
}

\author{
Rajesh Jayaraman ${ }^{1, *}$, Sham Sunder ${ }^{1}$, Vijay Kumar Gupta², Neera Sharma ${ }^{3}$, Satyanand Sathi ${ }^{1}$, Himanshu Mahapatra ${ }^{1}$, Venkatara- \\ mana Krishnamurthy ${ }^{1}$, Himanshu Verma ${ }^{1}$, Prabhu Kanchi ${ }^{1}$, Anurag Gupta ${ }^{1}$, Pranit Ram ${ }^{1}$, and Sunil Kumar Daksh ${ }^{1}$
}

${ }^{1}$ Department of Nephrology, Ram Manohar Lohia Hospital, New Delhi, India

${ }^{2}$ Department of Cardio-Thoracic Surgery, Ram Manohar Lohia Hospital, New Delhi, India

${ }^{3}$ Department of Biochemistry, Ram Manohar Lohia Hospital, New Delhi, India

*Corresponding author: Rajesh Jayaraman, Post Graduate, Department of Nephrology, Ram Manohar Lohia Hospital, New Delhi, India, Phone: 82482 11454; E-mail: rajeshumadevijayaraman@gmail.com

Received: 18 Apr, 2020 | Accepted: 04 May, 2020 | Published: 08 May, 2020

Citation: Jayaraman R, Sunder S, Gupta VK, Sharma N, Sathi S, et al. (2020) The Ability of Novel Urinary Biomarkers (NGAL and IL-18) in Predicting the in-Hospital Mortality, the Need of Renal Replacement Therapy and the Future Development of Chronic Kidney Disease in Post-Cardiac Surgery Acute Kidney Injury Patients: A New Horizon of Hope on the Anvil. Int J Nephrol Kidney Fail 6(2): dx.doi.org/10.16966/23805498.194

Copyright: (c) 2020 Jayaraman R, et al. This is an open-access article distributed under the terms of the Creative Commons Attribution License, which permits unrestricted use, distribution, and reproduction in any medium, provided the original author and source are credited.

\begin{abstract}
Background: Novel urinary biomarkers have always remained/continue to remain a fascinating area of exploration in the domain of modern nephrology. They are promisingly convincing in obviating the major disadvantages of creatinine in the setting of Acute Kidney Injury (AKI). The specific novel tubular markers include Neutrophil Gelatinase Associated Lipocalin (NGAL), Interleukin-18 (IL-18), Kidney Injury Molecule-1 (KIM-1), Hepatocyte growth factor, Osteopontin and Fatty acid binding proteins.
\end{abstract}

Methods: Hundred patients who underwent cardiac surgery in our institution were enrolled. The pattern of AKI including its incidence, the performance of the biomarkers NGAL and IL-18 in predicting the requirement of Renal Replacement Therapy (RRT), the in-hospital mortality and the future development of Chronic Kidney Disease (CKD) were all studied.

Prospective patients above 18 years of age undergoing elective cardiac surgery, those without evidence of pre-existing CKD and without AKI were included. Statistical analysis was performed using STATA 11.0 (Stata Corp LP, College Station, TX, USA). Shapiro-Wilk test was applied to test for normality of continuous variables. Categorical data were analysed using chi-square test.

Results: Both adjusted (adjusted for urinary creatinine) and unadjusted NGAL at 7 days post-operative predicted the in-hospital mortality perfectly. A $7^{\text {th }}$ day adjusted NGAL value of $808.46 \mathrm{pg} / \mathrm{mg}$ of creatinine was probably the best predictor of in-hospital mortality. An adjusted $7^{\text {th }}$ day IL-18 of $>704.45 \mathrm{pg} / \mathrm{ml}$ predicted a mortality risk close to that observed with NGAL. Urinary NGAL of $650 \mathrm{pg} / \mathrm{ml}$ predicted the need of RRT to $85 \%$. Urinary IL-18 was not a reliable predictor of RRT. Both the biomarkers predicted the future development of CKD reliably. Serum creatinine was inferior to both in predicting the in-hospital mortality and the need of RRT.

Conclusions: Raised urinary biomarkers NGAL, IL-18 in cardiac surgery patients are reliable indicators of predicting the in-hospital mortality the need of RRT and the future development of CKD than serum creatinine in post-cardiac surgery AKI patients.

Keywords: Post-Cardiac Surgery; Acute Kidney Injury; NGAL; IL-18

\section{Introduction}

Acute kidney injury (AKI) is a common and serious complication of cardiothoracic surgery [1]. Depending on the definition of AKI used, the incidence may be as low as $7.7 \%$ to as high as $28 \%$, necessitating RRT in $1 \%$ to $5 \%$ [2-9]. In our study, we found an incidence of $31 \%$. In order to recapitulate the basic physiology of NGAL and IL-18, their roles and function at the molecular level, the readers are requested to have a glimpse on the background section of the first half of our study. Our research article embraced six broader ranges of objectives as follows. 
The incidence of AKI in post-cardiac surgery patients in our institution. The ability of both the biomarkers in detecting AKI early when compared to serum creatinine. Their precision in differentiating pre-renal from intrinsic AKI. Their efficiency in predicting the need of RRT, the in-hospital mortality and the future development of CKD over subsequent follow-ups.

Our present study emphasises the role of both the biomarkers in predicting the in-hospital mortality after cardiac surgery as the main objective with their efficacy in predicting the need of RRT and the future development of CKD as the secondary objectives.

\section{Subjects and Methods}

This is an open-labelled non-randomized prospective observational study lasting for 18 months from the time of enrolling the patients to the completion of the study. 100 patients were selected without any bias who got admitted to the cardiothoracic surgery department in Ram Manohar Lohia Hospital, New Delhi, India to undergo elective cardiac surgery between January 2012 and June 2013. Informed consent was obtained from all patients included in the study. Elective cardiac surgeries include Coronary Artery Bypass Grafting (CABG) both on and off pump, valve repairs, mitral and aortic valve replacements and atrial and ventricular septal defect closures.

\section{Inclusion and exclusion criteria, clinical/laboratory parame- ters, the method of estimation of urinary biomarkers and the details of the follow-up}

All the patients who took part in the study met the following inclusion criteria:

a) Prospective patients above 18 years of age undergoing elective cardiac surgery.

b) Patients who did not have any evidence of pre-existing CKD as explained by normal kidney size and echogenic pattern in ultrasound kidney-ureter-bladder.

c) Patients with normal pre-operative baseline blood urea and serum creatinine.

d) Patients with no documented evidence of any AKI or recovery from AKI in the past.

The following patients were excluded from the study:

Patient's $\leq 18$ years of age, post renal transplant patients, patients with any past evidence of CKD including chronic proteinuria, ultrasonographic evidence of shrunken kidneys and altered echogenicity, deranged baseline renal function, patients with any documented evidence of past AKI or recovery from AKI were excluded from the study.

Patients were evaluated at 3 definite pre-determined time points of their hospital stay, including pre-operatively on the morning of the day of surgery, 24 hours after completion of the surgery, and 7 days after completion of the surgery.

Following were the patient's characteristics and parameters estimated on the morning of the day of surgery, 24 hours and 7 days after surgery: Demographic details, past medications, baseline creatinine in $\mathrm{mg} / \mathrm{dL}$, urine output in $\mathrm{mL} / \mathrm{h}, 24$ hours urinary creatinine in $\mathrm{mg} / \mathrm{dL}$, urinary NGAL in $\mathrm{pg} / \mathrm{mL}$, urinary NGAL adjusted to urinary creatinine in $\mathrm{pg} / \mathrm{mg}$ of creatinine, urinary interleukin- $18 \mathrm{in} \mathrm{pg} / \mathrm{mL}$ and also adjusted to urinary creatinine in $\mathrm{pg} / \mathrm{mg}$ of creatinine, and fractional excretion of sodium and urea (FENa and FEurea).

Glomerular Filtration Rate (GFR) was measured as per 4-variable modification of diet in renal diseases (MDRD) formula. Both FENa and FEurea were used to differentiate pre renal from intrinsic AKI. Biomarkers' values were expressed in unadjusted forms and adjusted to urinary creatinine to correct for variations in urine flow. The pre-operative values were considered the baseline values of the patients. Fresh midstream urine samples were collected for the measurement of the biomarkers and urinary creatinine. Biomarkers were measured by ELISA using BIORAD ELISA kits. Hycult biotech-HK 330 human NGAL ELISA kit was used for urinary NGAL measurement and IL-18 was measured using MBL human IL-18 ELISA kit. Urinary NGAL of $<60 \mathrm{pg} / \mathrm{mL}$ and IL- 18 of $<257 \mathrm{pg} / \mathrm{mL}$ was considered as normal as per the standardization provided by the laboratory kits. Both the markers were adjusted with urinary creatinine and compared with their unadjusted parameters in the study. Plasma and urine creatinine were measured by alkaline picrate reaction, blood urea by urease methodology and all the other routine investigations were done by "automated clinical chemistry analyser". Persons' who did not develop AKI were taken as controls.

In addition, all those 31 individuals who developed AKI during the study were followed-up for at least 3 months and beyond depending upon their compliance. Serum creatinine, blood urea and all the other basic parameters were estimated serially after developing AKI, at the end of three months and periodically after that to see for the development of CKD and its progression, if any (Figure 1).

\section{Statistical analysis}

Statistical analysis was performed using STATA 11.0 (Stata Corp LP, College Station, TX, USA). Shapiro-Wilk test was applied to test for normality of continuous variables. Parametric data were described as mean \pm standard deviation. Median and Inter-quartile Range (IQR) were used to describe nonparametric data. Mann-Whitney test was employed to compare the non-parametric data between two groups. Categorical data were analysed using chi-square test. A P-value of $<0.05$ was considered statistically significant. Logistic regression was employed to predict the categorical outcome from the independent variables. Area under curve (AUC) was utilized to determine at point of different parameters to distinguish between the subjects in AKI and normal (control) group.

\section{Observations and Results}

\section{Biomarkers' predictability regarding the in-hospital mor- tality}

The in-hospital mortality and the co-existent organ dysfunction were mainly assessed through APACHE II scoring which employs varied parameters for different organ dysfunctions. The rising score was compared with the level of the biomarkers and graphs were plotted accordingly. A positive correlation was found between the rising APACHE II scores and rising levels of both the adjusted and unadjusted NGAL and IL-18. Median APACHE-II score in AKI patients was 12.37 (IQR, 10 to 16.4) and 8 for controls (IQR, 7.3 to 9). The observed difference was statistically significant; $\mathrm{p}<0.0001$, Mann-Whitney test. The median duration of ICU stay was 4 days for both AKI and controls with an IQR of (3-7) for the former and 3.5 to 5 for the latter. Both were not statistically different. ( $\mathrm{p}=0.42$, Mann-Whitney test) (Figure 2 ).

NGAL and IL-18 were tested individually and in combination for their ability to predict the overall mortality in patients with AKI in our study. Mortality consistently increased with unadjusted $24 \mathrm{hr}$ NGAL level of $>50 \mathrm{pg} / \mathrm{mL}$ and with $>500 \mathrm{pg} / \mathrm{mL}$ with a mortality of $60 \%$. Adjusted NGAL at $24 \mathrm{hrs}$ gave more refined results. A value of $1800-$ $1900 \mathrm{pg} / \mathrm{mg}$ of creatinine was associated with $90 \%$ in-hospital mortality (Figures 3 and 4 ). 


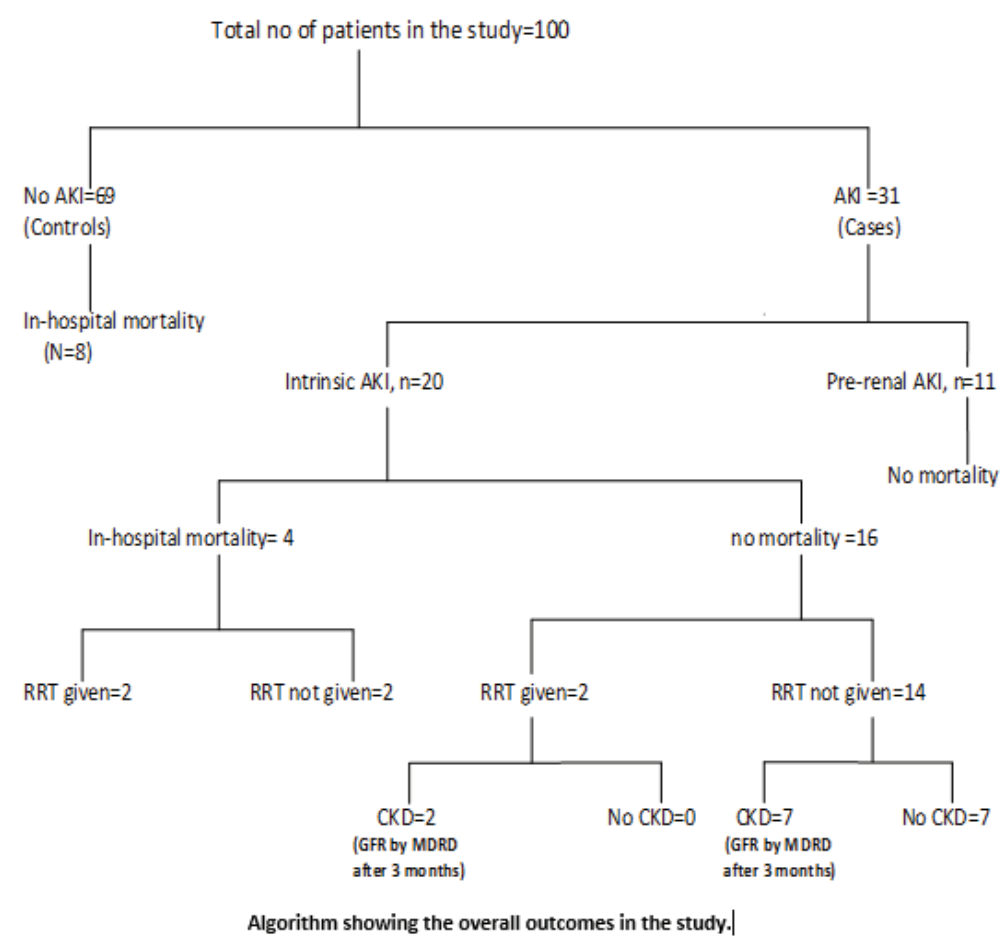

Figure 1: Algorithm showing the outcomes of our study in a nutshell.

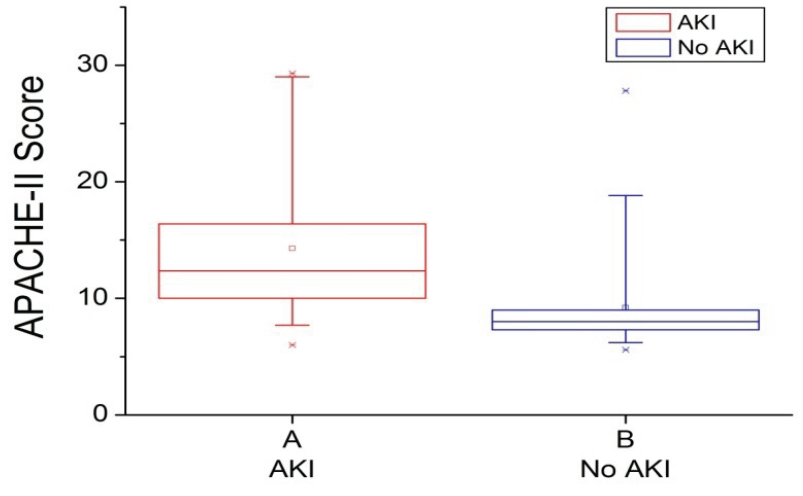

Figure 2: Box-whisker plot showing APACHE II score among cases and controls.

The risk of in-hospital mortality increased with $24 \mathrm{hr}$ IL-18 of $>250 \mathrm{pg} / \mathrm{mL}$. With a value of $400 \mathrm{pg} / \mathrm{ml}$, the mortality was $60 \%$ and with a value of $>550 \mathrm{pg} / \mathrm{mL}$, it was up to $85 \%$.

Both adjusted and unadjusted NGAL at 7 days post-op predicted the in-hospital mortality perfectly. A $7^{\text {th }}$ day adjusted NGAL value of $808.46 \mathrm{pg} / \mathrm{mg}$ of creatinine was probably the best predictor of in-hospital mortality among all the parameters observed in the study.

Similarly, a persistently elevation by the $7^{\text {th }}$ day IL- 18 consistently predicted an increased mortality. A value of $>350 \mathrm{pg} / \mathrm{mL}$ predicted $40 \%$ mortality while $450 \mathrm{pg} / \mathrm{mL}$ and $600 \mathrm{pg} / \mathrm{mL}$ predicted a mortality approximately $65 \%$ and $80 \%$, respectively. An adjusted $7^{\text {th }}$ day IL-18 of $>704.45 \mathrm{pg} / \mathrm{mL}$ predicted a mortality risk close to that observed with NGAL (Figure 5).

Mortality prediction by creatinine on the $7^{\text {th }}$ post-operative day was found to be inferior to the other two markers, with a creatinine of 2.3$2.4 \mathrm{mg} \%$ predicting a mortality of $15 \%$ and a creatinine of $4.2 \mathrm{mg} \%$, a mortality of $38 \%$. This could be considered as a bias created by the initiation of RRT that reduces the serum creatinine level rather than the poor performance of creatinine itself, as 3 out of 4 patients with AKI who expired in ICU were dialysis dependent. $7^{\text {th }}$ day creatinine was also compared with the biomarker levels in those who developed CKD as per the estimated GFR during the 3 months of follow-up.

\section{Prediction of the need of RRT by the biomarkers}

$24 \mathrm{hr}$ NGAL was a reliable predictor of the need for RRT. Beyond a value of $100 \mathrm{pg} / \mathrm{mL}$, the probability for requirement of RRT consistently increased. At a value $500 \mathrm{pg} / \mathrm{mL}$, it was $60 \%$ and with a value of $600-650 \mathrm{pg} / \mathrm{mL}$, the risk went up to $85 \%$. An adjusted NGAL was also found to be a perfect predictor of RRT in post-cardiac surgery patients. $24 \mathrm{hr}$ IL-18 was not a significant predictor of RRT ( $\mathrm{p}=0.23$, logistic regression). $24 \mathrm{hr}$ NGAL + IL-18 predicts RRT perfectly. The combination was not found to be superior to NGAL alone. While adjusted NGAL gave the same significance as its unadjusted counterpart, adjusted IL-18 was not a significant predictor of RRT ( $\mathrm{p}=0.49$, logistic regression).

The probability of predicting RRT based on the rise in serum creatinine at $24 \mathrm{hrs}$ was found to be low, being around $18 \%$ when creatinine was between 1.4 and 1.8. This low probability in part might be attributed to the inability of creatinine to rise in the serum at $24 \mathrm{hrs}$ of AKI as did the other two urinary biomarkers (Figure 6). 


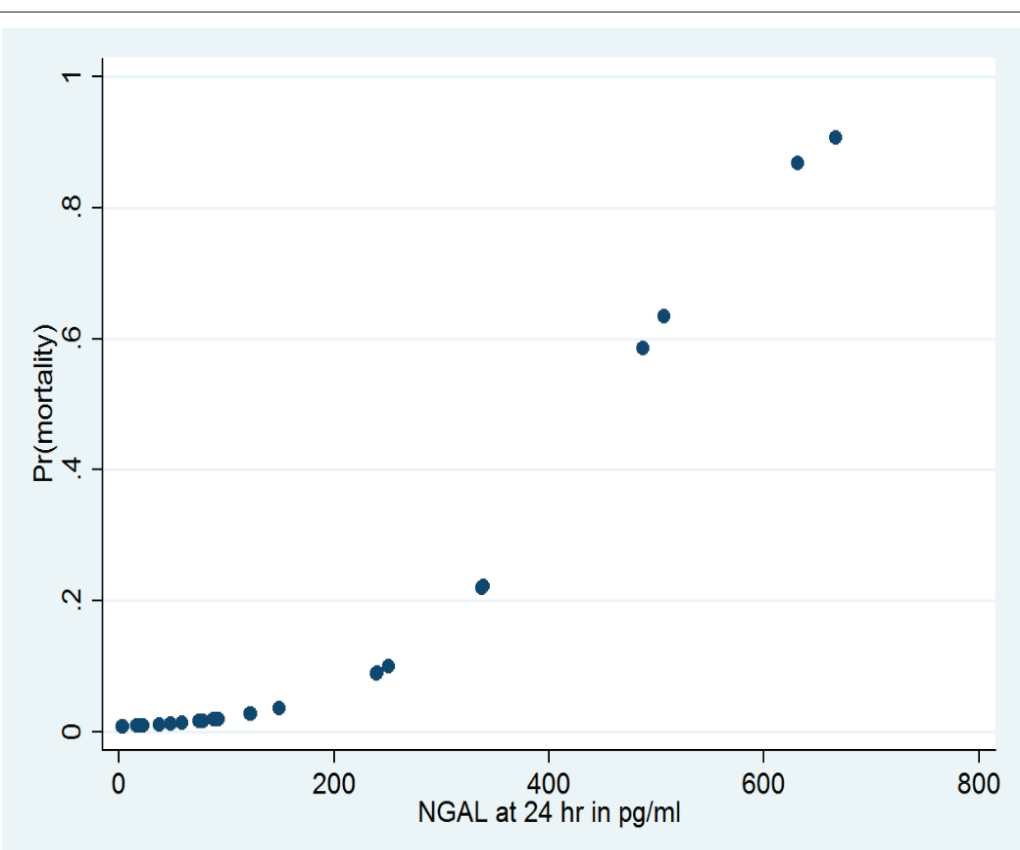

Figure 3: Scatter-plot showing $24 \mathrm{hr}$ unadjusted NGAL and mortality prediction.

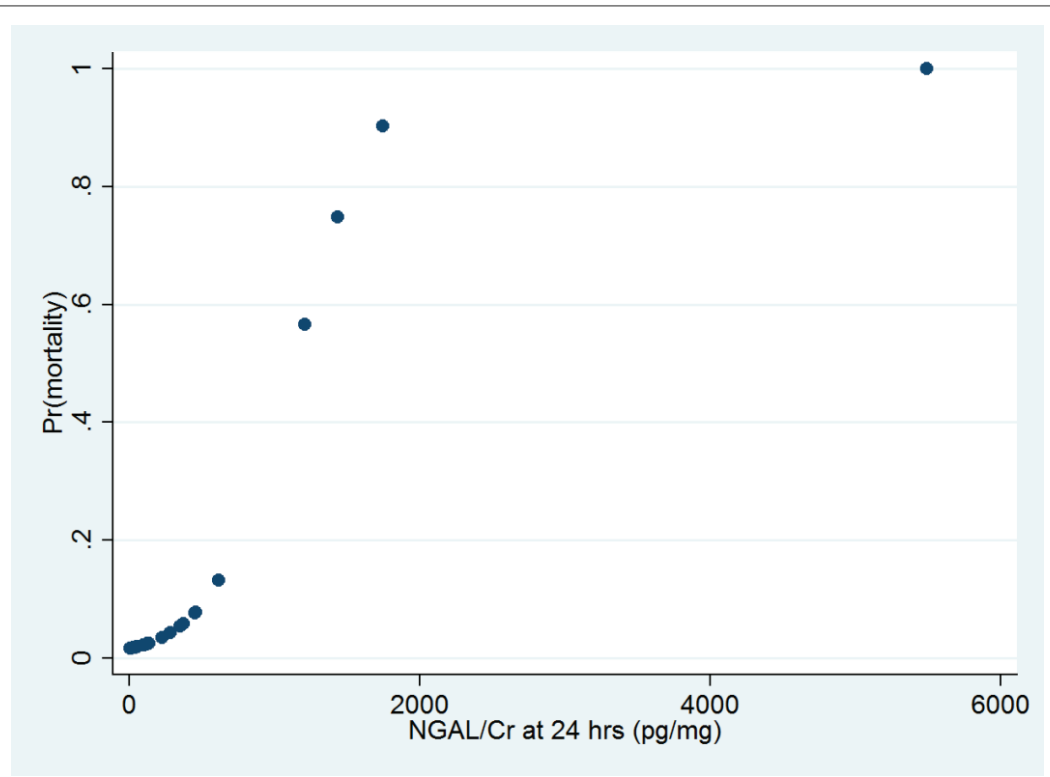

Figure 4: Scatter-plot showing $24 \mathrm{hr}$ adjusted NGAL and its relation to mortality.

$24 \mathrm{hr}$ IL-18 was not a significant predictor of RRT ( $\mathrm{p}=0.23$, logistic regression). $24 \mathrm{hr}$ NGAL + IL-18 predicted RRT perfectly. The combination was not found to be superior to NGAL alone. Both the adjusted and unadjusted NGAL provided the same level of significance (Figure 7).

\section{Biomarkers' ability to predict the future development of CKD in those with AKI}

An unadjusted and adjusted 7th day NGAL of $>49.6 \mathrm{pg} / \mathrm{mL}$ and $>68.21 \mathrm{pg} / \mathrm{mg}$ were probably the best predictors for the development of CKD followed by adjusted and unadjusted IL-18. An adjusted $7^{\text {th }}$ day IL-18 of 520pg/mL and an unadjusted value of $400 \mathrm{pg} / \mathrm{mL}$ predicted the development of CKD close to that of NGAL (Figure 8).

\section{Discussion}

Novel biomarkers namely NGAL and IL-18 have thoroughly transformed the domain of investigative nephrology including early diagnosis, assessing severity and in predicting the prognosis in various forms of AKI.

In a study done by Bennett M, et al. [10], AKI developed in 99 of 196 patients in whom a diagnosis due to creatinine was delayed up to 3 days after CPB. In contrast, mean urine NGAL levels increased 15 -fold within $2 \mathrm{~h}$ and by 25 -fold at 4 and $6 \mathrm{~h}$ after CPB. The 2 -h urine NGAL levels correlated with severity and duration of AKI, length of stay, dialysis requirement, and death. 


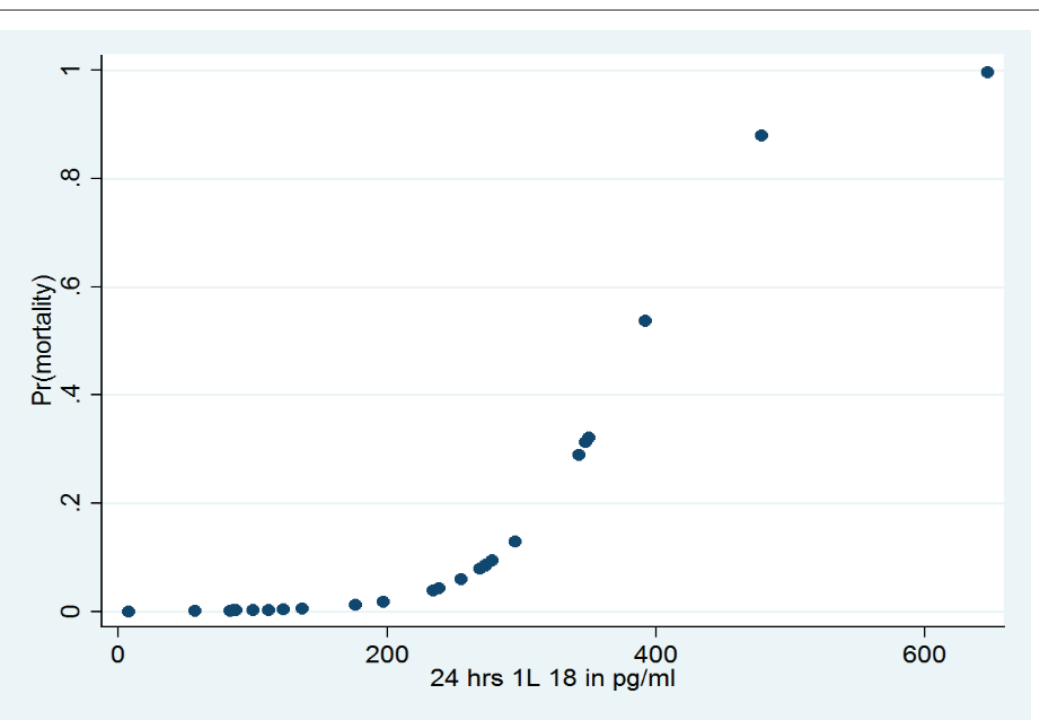

Figure 5: $24 \mathrm{hr}$ urinary IL-18 and its role in mortality prediction.

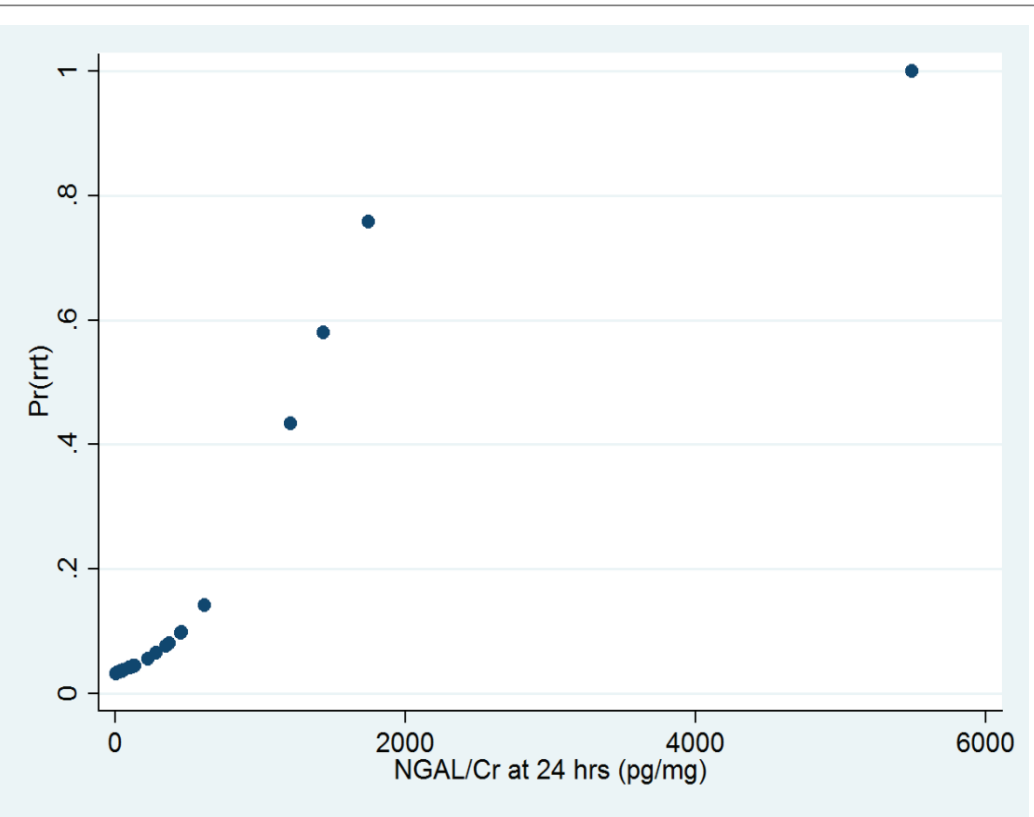

Figure 6: Adjusted NGAL and RRT prediction.

In various studies [11-17] done on this biomarker, Urine NGAL peaked immediately after cardiac surgery and remained significantly higher 3, 18 and $24 \mathrm{~h}$ after surgery. Two hours postoperative plasma NGAL strongly correlated with changes in creatinine, duration of AKI, length of hospital stays and mortality.

Pezeshgi A, et al. [18] in their recent study couldn't establish the ability of urinary NGAL in diagnosing AKI early and in the prediction of mortality among the observed post coronary artery bypass patients. The obvious reason for the underperformance of the novel biomarker would be the low sample size taken into consideration in their study.

In our study, rising urinary NGAL levels were very well correlated with raising APACH II scoring, in-hospital mortality and requirement of RRT. A positive correlation was found between both adjusted and unadjusted NGAL and raising APACHE II score through linear re- gression. In-hospital mortality consistently increased with unadjusted $24 \mathrm{hr}$ NGAL level of $>50 \mathrm{pg} / \mathrm{mL}$ and with a $24 \mathrm{hr}$ value of $>500 \mathrm{pg} / \mathrm{mL}$, the mortality was over $60 \%$. An adjusted NGAL value of $1800-1900$ $\mathrm{pg} / \mathrm{mg}$ of creatinine at $24 \mathrm{hrs}$ was associated with $90 \%$ in-hospital mortality. A $7^{\text {th }}$ day adjusted NGAL value of $808.46 \mathrm{pg} / \mathrm{mg}$ of creatinine was probably the best predictor of in-hospital mortality.

To the best of our knowledge, ours is probably the first study to predict the increased risk of CKD in those post-cardiac surgery patients with $\mathrm{AKI}$ whose seventh day urinary biomarker levels remained very high. An unadjusted $7^{\text {th }}$ day NGAL of $>49.6 \mathrm{pg} / \mathrm{mL}$ and an adjusted $7^{\text {th }}$ day NGAL/Cr $>68.21 \mathrm{pg} / \mathrm{mg}$ best predicted the development of CKD.

Lobato GR, et al. [19] in their exploration found that Urinary NGAL was predictive of CKD progression, end stage renal disease and death in non-dialyzed patients and was associated with an increased risk of 


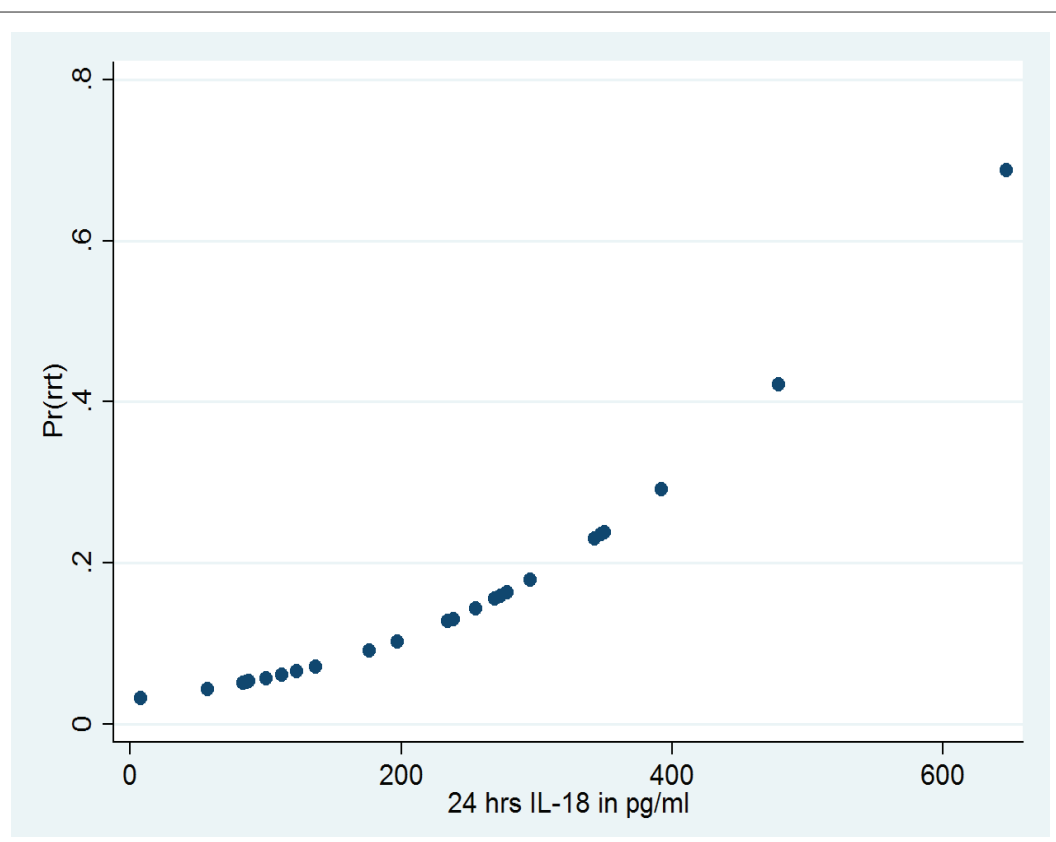

Figure 7: IL-18 and its RRT prediction.

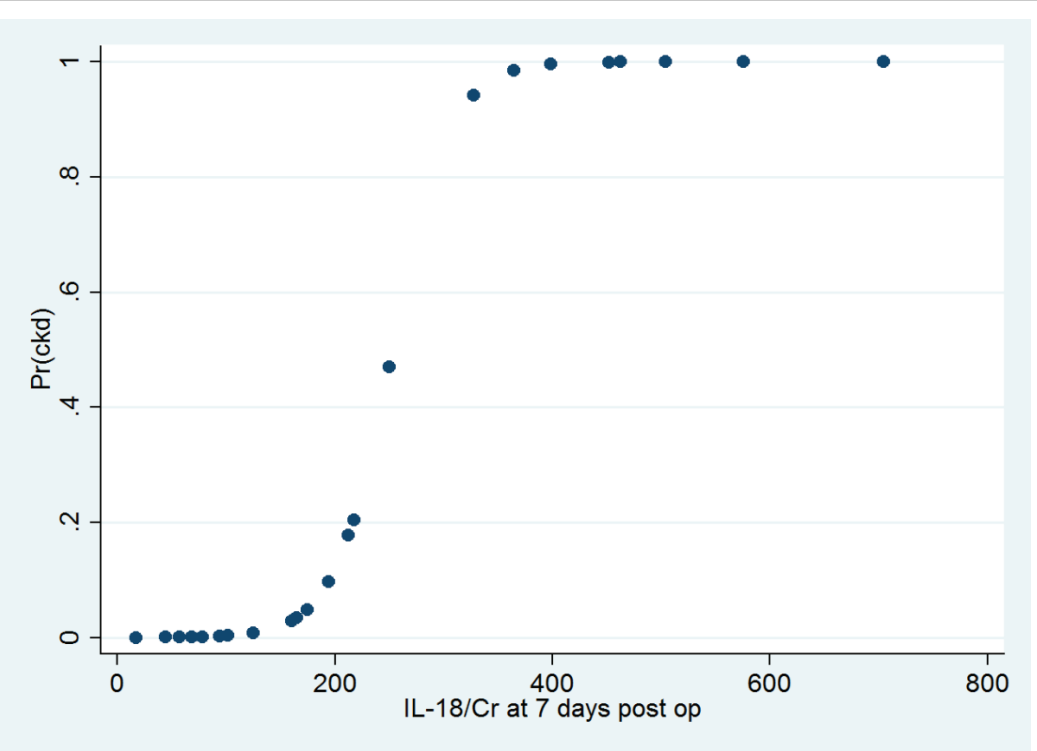

Figure 8: Adjusted urinary IL-18 and CKD prediction.

death in patients on dialysis. But here, the study cohort comprised of CKD patients in their early stages and not AKI.

\section{Conclusions}

The novel urinary biomarkers NGAL, IL-18 have been a breakthrough in many AKI states. In post-cardiac surgery patients, they are effective not only in early detection of AKI but their quantitative measure can precisely predict the need for RRT in the ICU settings, the in-hospital mortality and the future risk of developing CKD. Though NGAL and IL-18 appear promising in obviating various known demerits of creatinine, they do not have a worldwide distribution, which limits their application. Only sites with economic privilege have access to them.

\section{Conflict of Interest}

None

\section{References}

1. Wijeysundera DN, Karkouti K, Dupuis JY, Rao V, Chan CT, et al. (2007) Derivation and validation of a simplified predictive index for renal replacement therapy after cardiac surgery. JAMA 297: 1801-1809.

2. Abel RM, Buckley MJ, Austen WG, Barnett GO, Beck CH Jr, et al. (1976) Etiology, incidence, and prognosis of renal failure following cardiac operations. Results of a prospective analysis of 500 consecutive patients. J Thorac Cardiovasc Surg 71: 323-333. 
3. Andersson LG, Ekroth R, Bratteby LE, Hallhagen S, Wesslen O (1993) Acute renal failure after coronary surgery--a study of incidence and risk factors in 2009 consecutive patients. Thorac Cardiovasc Surg 41: 237-241.

4. Chertow GM, Levy EM, Hammermeister KE, Grover F, Daley J (1998) Independent association between acute renal failure and mortality following cardiac surgery. Am J Med 104: 343-348.

5. Conlon PJ, Stafford-Smith M, White WD, Newman MF, King S, et al. (1999) Acute renal failure following cardiac surgery. Nephrol Dial Transplant 14: 1158-1162.

6. Mangano CM, Diamondstone LS, Ramsay JG, Aggarwal A, Herskowitz A, et al. (1998) Renal dysfunction after myocardial revascularization: risk factors, adverse outcomes, and hospital resource utilization. The Multicenter Study of Perioperative Ischemia Research Group. Ann Intern Med 128: 194-203.

7. Ostermann ME, Taube D, Morgan CJ, Evans TW (2000) Acute renal failure following cardiopulmonary bypass: a changing picture. Intensive Care Med 26: 565-571.

8. Tuttle KR, Worrall NK, Dahlstrom LR, Nandagopal R, Kausz AT, et al. (2003) Predictors of ARF after cardiac surgical procedures. Am J Kidney Dis 41: 76-83.

9. Zanardo G, Michielon P, Paccagnella A, Rosi P, Calo M, et al. (1994) Acute renal failure in the patient undergoing cardiac operation. Prevalence, mortality rate, and main risk factors. J Thorac Cardiovasc Surg 107: 1489-1495.

10. Bennett $\mathrm{M}$, Dent $\mathrm{CL}, \mathrm{Ma} \mathrm{Q}$, Dastrala S, Grenier F, et al. (2008) Urine NGAL predicts severity of acute kidney injury after cardiac surgery: a prospective study. Clin J Am Soc Nephrol 3: 665-673.

11. Wagener G, Jan M, Kim M, Mori K, Barasch JM, et al. (2006) Association between increases in urinary neutrophil gelatinaseassociated lipocalin and acute renal dysfunction after adult cardiac surgery. Anesthesiology 105: 485-491.
12. Haase-Fielitz A, Bellomo R, Devarajan P, Story D, Matalanis G, et al. (2009) Novel and conventional serum biomarkers predicting acute kidney injury in adult cardiac surgery--a prospective cohort study. Crit Care Med 37: 553-560.

13. Haase M, Bellomo R, Devarajan P, Ma Q, Bennett MR, et al. (2009) Novel biomarkers early predicts the severity of acute kidney injury after cardiac surgery in adults. Ann Thorac Surg 88: 124-130.

14. Haase-Fielitz A, Bellomo R, Devarajan P, Bennett M, Story D, et al. (2009) The predictive performance of plasma neutrophil gelatinaseassociated lipocalin (NGAL) increases with grade of acute kidney injury. Nephrol Dial Transplant 24: 3349-3354.

15. Tuladhar SM, Püntmann VO, Soni M, Punjabi PP, Bogle RG, et al. (2009) Rapid detection of acute kidney injury by plasma and urinary neutrophil gelatinase-associated lipocalin after cardiopulmonary bypass. J Cardiovasc Pharmacol 53: 261-266.

16. Capuano F, Goracci M, Luciani R, Gentile G, Roscitano A, et al. (2009) Neutrophil gelatinase-associated lipocalin levels after use of minicardiopulmonary bypass system. Interact Cardiovasc Thorac Surg 9 797-801.

17. Wagener G, Gubitosa G, Wang S, Borregaard N, Kim M, et al. (2009) A comparison of urinary neutrophil gelatinase-associated lipocalin in patients undergoing on- versus off-pump coronary artery bypass graft surgery. J Cardiothorac Vasc Anesthesia 23: 195-199.

18. Pezeshgi A, Behmanesh A, Esmaeilzadeh A, Chiti H, Kamali K, et al. (2020) Can urinary neutrophil gelatinase-associated lipocalin have a role in the early diagnosis of acute kidney injury after coronary artery bypass graft? J Renal Inj Prev 9.

19. Lobato GR, Lobato MR, Thomé FS, Veronese FV (2017) Performance of urinary kidney injury molecule-1, neutrophil gelatinaseassociated lipocalin, and $\mathrm{N}$-acetyl- $\beta$-D-glucosaminidase to predict chronic kidney disease progression and adverse outcomes. Braz J Med Biol Res 50: e6106. 